\title{
Atherosclerotic Cardiovascular Disease
}

National Cancer Institute

\section{Source}

National Cancer Institute. Atherosclerotic Cardiovascular Disease. NCI Thesaurus. Code C35771.

Cardiovascular disease resulting from atherosclerosis. 